\title{
Development of the Female Reproductive Tract and Associated Anomalies
}

\author{
Janeen L. Arbuckle, MD, PhD* \\ Kimberly H. Hoover, MD
}

\author{
Address \\ *176 F, 10261, 619 19th Street South, Birmingham, AL, 35249, USA \\ Email: jarbuckle@uabmc.edu
}

Published online: 1 September 2016

C) Springer International Publishing AG 2016

\section{This article is part of the Topical Collection on Pediatric Gynecology}

Keywords Female reproductive tract · Aberrations - Anomalies $\cdot$ Reproductive organs

\section{Opinion statement}

Aberrations in the development of the female reproductive tract result in an array of anomalies. Because of the wide range of possible anomalies, the presentation of women with congenital anomalies of the reproductive tract is highly variable. While some women are asymptomatic, others present with symptoms of pain, menstrual abnormality, recurrent pregnancy loss, and/or poor obstetric outcomes. When providing care for patients with anomalies of the female reproductive tract, it is critical to be sensitive to both the patient's physical and psychological well-being. When an intervention is indicated, patient maturity, motivation, and ability to be an active participant in their care should be considered. Due to the rarity of some of the anomalies of the female reproductive tract, care at a center with special expertise in the care of these complex patients is at times warranted.

\section{Introduction}

\section{The embryological development of the female reproductive organs}

The development of the reproductive system begins at approximately 6 weeks gestational age. Initially the system consists of undifferentiated gonads and two paired ductal systems. The two ductal systems are the Wolffian ducts and the Müllerian ducts which represent the precursors to the male and female reproductive systems, respectively. Though gonads influence the development of the reproductive organs, they have different embryologic origins and are typically unaffected in patients with Müllerian anomalies.

In males, the developing testes secrete two substances that influence the development of the male reproductive tract. The first substance is Müllerian Inhibiting Substance (MIS) which causes the Müllerian ducts to regress. The testes also produce testosterone which promotes Wolffian differentiation into the components of the male reproductive system. 
In females, neither MIS nor testosterone is produced. The Wolffian ducts therefore regress while the Müllerian ducts persist. The Müllerian ducts extend in a cranialcaudal fashion and fuse at their caudal end to form a yshaped structure, the uterovaginal primordium. This medial fusion event creates a septum which will subsequently regress, resulting in the formation of the uterus, the cervix, and the upper portion of the vagina. The cranial, unfused portions of the Müllerian ducts will become the paired fallopian tubes.

The caudal portion of the uterovaginal primordium fuses with the urogenital sinus, canalization of which results in the formation of the vagina and hymen. A portion of the vagina has traditionally been thought to be derived from the urogenital sinus. There is, however, significant debate with regard to the embryologic origins of the vagina. Regardless of its origins, the vagina's development is dependent on the concomitant development of the Müllerian ducts and estrogen.

At birth, the female reproductive system is comprised of paired ovaries and fallopian tubes, a single uterine body and cervix, and a single vagina. Aberrations in the embryological development of the female reproductive tract may result in any one of several different anomalies. The development of the female reproductive tract is closely linked to the developing renal system, and patients with Müllerian anomalies often have associated renal anomalies $[1,2]$.

\section{Classification of anomalies of the female reproductive tract}

Classification of anomalies of the female reproductive tract has been the subject of recent debate. The American Society of Reproductive Medicine (ASRM, formally American Fertility Society) first developed a classification system to describe anomalies of the Müllerian system in 1979. This was expanded in 1988 and is the primary system for classification of anomalies of the female reproductive tract [3]. Alternate classification systems such as the Vagina Cervix Uterus Adnexa-associated Malformation (VCUAM) system [4], the European Society of Human Reproduction and Embryology (ESHRE), and the European Society for Gynecological Endoscopy (ESGE) have also been proposed [5, 6]. Though each of these systems has its own merits, for ease of discussion here, we will broadly discuss the anomalies of the reproductive tracts as they fall into one of three categories: (1) uterine aplasia, (2) obstructive anomalies, or (3) non-obstructive anomalies (summarized in Table 1).

\section{Diagnosis and imaging}

In females in whom a Müllerian anomaly is suspected, ultrasound is commonly the first imaging modality utilized to delineate pelvic structures. Though transvaginal ultrasound is the preferred means of imaging the female pelvis, this approach is not always feasible or tolerated in the pediatric and adolescent population. For these patients, transabdominal and translabial images can be helpful. Though the vagina and cervix are not well visualized using ultrasound, the uterine and ovarian anatomy can be well described using this imaging modality. Imaging is enhanced with the use of $3 \mathrm{D}$ ultrasound which can improve the accuracy in characterization of Müllerian anomalies to approximately $90 \%$.

When ultrasound alone is not sufficient to fully delineate the Müllerian anomaly, MRI is the imaging modality of choice and is considered the gold standard in imaging the female pelvis [8]. Furthermore, MRI may provide additional information of the renal system abnormalities of which are commonly associated with Müllerian anomalies.[7]

\section{Uterine aplasia}

- Mayer-Rokitansky-Küster-Hauser (MRKH)

- Complete Androgen Insensitivity Syndrome (CAIS)

Uterine aplasia, seen in Mayer-Rokitansky-Küster-Hauser (MRKH) syndrome and disorders of sexual differentiation such as androgen insensitivity syndrome (AIS), typically presents in adolescence with 


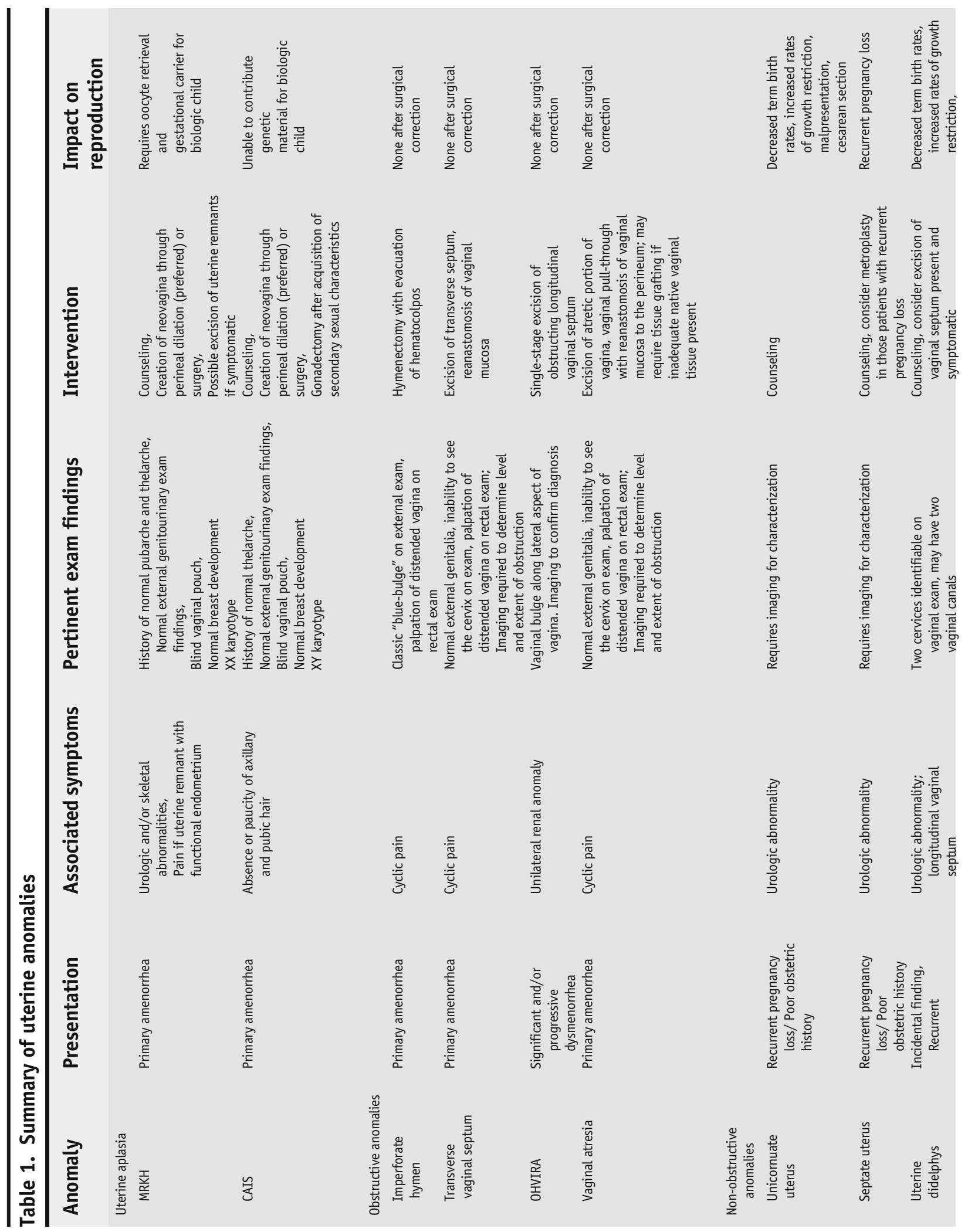




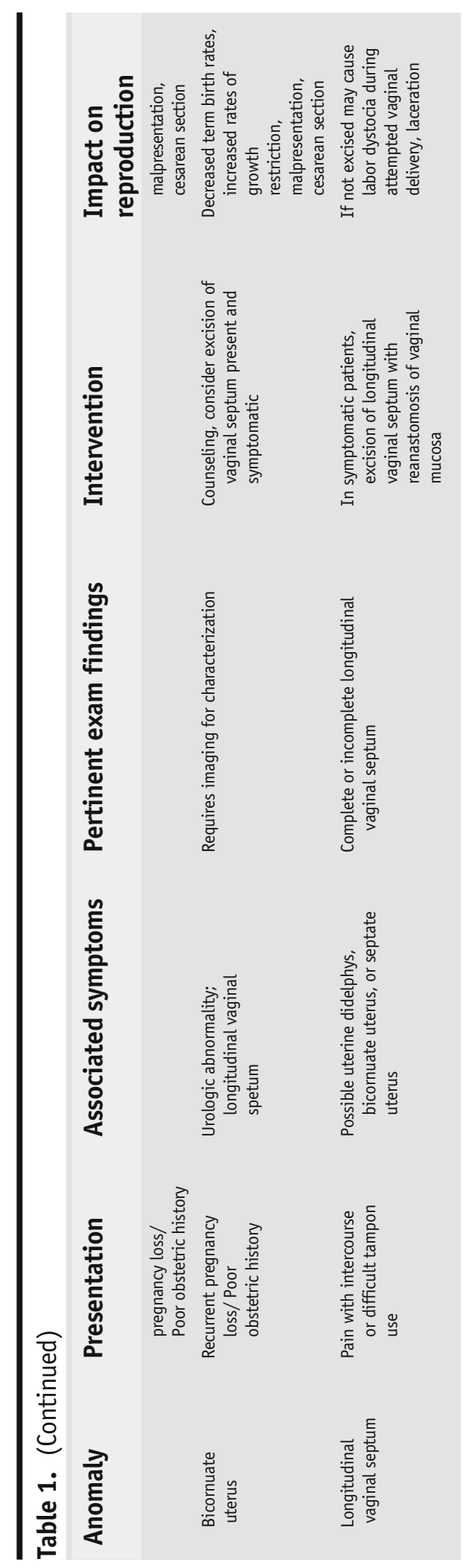




\section{MRKH}

primary amenorrhea. External exam of these patients will reveal normal external genitalia with either a blind vaginal pouch or a vaginal dimple. Imaging is then utilized to assess for the presence or absence of the uterus, cervix and upper vagina. When uterine aplasia is observed, it is important to further characterize the patient as the underlying syndrome will guide further management. A karyotype is essential to differentiating MRKH from AIS [9].

Uterine aplasia is most commonly associated with MRKH, affecting 1:40001:10,000 women [10]. The disorder occurs in females with an XX karyotype in whom the Müllerian ducts failed to develop, resulting in an absent vagina [11]. The ovaries, being of different embryologic origins than the Müllerian ducts, are unaffected. Affected females will report a history of normal female sexual maturation with thelarche and pubarche but no menarche. Though the majority of patients with MRKH have complete uterine aplasia, a number of patients will have fallopian tubes and/or rudimentary uterine horns. Though these uterine horns are typically non-functional, $2-7 \%$ of these rudimentary horns will have a functional endometrium and present with cyclic abdominal pain due to the obstruction of outflow [12]. Additional congenital anomalies, particularly of the urologic and skeletal systems, are observed in $25-50 \%$ of patients with MRKH $[10,11]$. As such, imaging of the renal system is also warranted when a diagnosis of MRKH is made.

\section{AIS}

AIS should also be considered in patients with uterine aplasia. AIS results from a defect in the androgen receptor (AR) and occurs with an incidence of 1:20,0001:99,000 genetic males $[13,14]$. AIS is inherited in an X-linked recessive pattern with variable presentations based on the inherited AR mutation. Those mutations which limit all signaling through the AR result in complete AIS (CAIS). CAIS is observed in XY individuals who, despite having normal testicular production of testosterone, develop a female phenotype. During embryogenesis in patient with CAIS, the Wolffian system is unresponsive to the circulating testosterone and does not differentiate into male structures. The developing testes also produce MIS which suppresses development of the Müllerian system, resulting in uterine aplasia. Testosterone serves as a precursor to estrogen which promotes thelarche in individuals with AIS at the time of puberty. Patients with CAIS will often not undergo pubarche and will have scant or absent pubic hair [14]. Due to the body's inability to respond to the effects of testosterone, individuals with CAIS typically identify as female, concordant with their phenotype [15]. Both partial and mild forms of AIS exist with varying effects on development.

\section{Treatment Options for Uterine Aplasia}

Treatment of the patient with uterine aplasia first begins with a consideration of the psychological impact of the diagnosis on the patient. As previously noted, uterine aplasia is commonly diagnosed in adolescence, a time of significant emotional flux. Understanding the diagnosis and addressing options for treatment are often distressing to adolescents and require a high degree of 
motivation. As these conversations will likely be difficult for the adolescent female, it is important to work closely with the patient, her family, and a counselor to allow the patient to best face her diagnosis $[15,16]$.

Once a patient is ready for intervention, attention is turned to the creation of a vagina. Though a number of surgical approaches have been described for the creation of a vaginal space, progressive perineal dilation is highly effective and is the preferred means of vaginal creation $[11,17,18]$. Several surgical approaches have been utilized for the creation of a neovagina and are reserved for those who have been unsuccessful at progressive vaginal dilation. These surgical procedures are described elsewhere and should be performed in a center with expertise with these procedures $[19,20]$. To achieve the best surgical outcome, patients should be prepared to participate with postoperative dilation or vaginal stenting. In those patients with pain related to a uterine remnant with functional endometrium, laparoscopic excision is warranted to mitigate symptoms.

Those patients who present with uterine aplasia due to CAIS are treated similarly to those with MRKH. Discordance of gender of identity and genetic sex can be psychologically distressing to the female with CAIS and counseling remains a key component of care. The majority of females with CAIS will identify as female [15] and will benefit from creation of a neovagina. In contrast to the MRKH patient, the patient with CAIS has testes which are at increased risk for germ cell tumors. Excision of the male gonad should be considered after the female has obtained secondary sexual characteristics. After gonadectomy, it is recommended for patients to continue supplemental estrogen with annual DEXA scans to evaluate bone health [21].

Uterine aplasia has a significant impact on a patient's reproductive options. Patients with MRKH have normal ovaries and may serve as an oocyte donor to achieve genetic offspring. Until recently, the only option for these patients was in vitro fertilization with the use of a gestational carrier. However, this requirement was recently bypassed in a patient with MRKH who delivered a live birth after uterine transplantation [22]. Though this procedure remains experimental, it may become a feasible option for women with uterine aplasia as the technology advances. Unfortunately, females with AIS do not maintain their reproductive potential.

\section{Obstructive anomalies of the female reproductive tract}

- Imperforate hymen

- Transverse vaginal septum

- Obstructive hemi-vagina with ipsilateral renal anomaly

- Vaginal atresia

Obstructive anomalies of the female reproductive tract often present in adolescence with a complaint of pain due to the obstruction of menstrual outflow. Obstruction may occur at any level of the outflow tract, ranging from the level of the hymen, as observed with imperforate 
hymen, to the cervix, as observed in cervical atresia. Definitive management requires surgical intervention, which, depending on the level of obstruction, may be simple or extensive. Those patients requiring extensive surgical procedures may not be emotionally or psychologically mature enough to participate in the postoperative management which often requires the use of a vaginal dilator. In patients who elect to delay surgical intervention, suppression of the menstrual cycle through hormonal suppression is a reasonable option. Suppression may be in the form of a combined oral contraceptive, progesterone injection, or with gonadotropin-releasing hormone agonists such as Depo-lupron [23]. Menstrual suppression prevents further accumulation of menstrual products and allows the patient time for emotional maturation until surgical intervention can be pursued.

The most common example of an obstructive anomaly is the imperforate hymen with an incidence of approximately 1:1000 [24]. Patients with imperforate hymen will present with primary amenorrhea and may report cyclic pain. On exam, there is a classic "blue-bulge" due to distension of the hymen. The presence of an imperforate hymen is further supported by having the patient Valsalva. The imperforate hymen, comprised of a thin layer of tissue, will bulge as the intra-abdominal pressure rises. Additional information may be obtained from rectal exam with a fullness felt immediately past the anal sphincter. If the diagnosis is still not certain after a thorough exam, ultrasound and/or MRI will help determine the diagnosis.

The imperforate hymen may also be observed in neonates, who, under the influence of maternal estrogen, develop vaginal secretions which accumulate and form a hydrocolpos and/or mucocolpos [20]. If the patient is asymptomatic, intervention may be delayed until puberty. If a significant accumulation has occurred, resulting in symptoms, excision of the hymen is warranted to allow for efflux of the accumulated fluid. Simple aspiration is contraindicated due to the risk of subsequent infection.

The treatment of the imperforate hymen is a cruciate incision of the hymen, allowing for efflux of menstrual fluid. This is typically performed during an exam under anesthesia, during which excess tissue may be excised as indicated [20]. Following the evacuation of the retained menstrual fluid, inspection of the vagina and cervix may also be performed. The hymen is derived from the urogenital sinus and, as such, is typically an isolated finding, without concomitant Müllerian or renal anomalies. Though rare, there have been case reports of associated anomalies detected at the time of treatment for an imperforate hymen [25-28].

Transverse vaginal septa are the rarest congenital anomaly with an incidence of 1:80,000 women [24]. A transverse vaginal septum arises either from incomplete vertical fusion of the Müllerian ducts and the urogenital sinus derivatives, or incomplete canalization of the fused structures. Therefore, patients with a transverse vaginal septum typically have normal upper tracts and renal systems [20]. 
Patients with a transverse vaginal septum typically present with primary amenorrhea. The septum is commonly located in the upper third of the vagina and may therefore not be readily apparent on external exam [29]. The transverse vaginal septum can vary in thickness and the surgical management and outcomes vary based on whether the septum is thin $(<1 \mathrm{~cm})$ or thick $(>1 \mathrm{~cm})$. In those individuals in whom a transverse septum is suspected, ultrasound or MRI may be utilized to characterize the patient's anatomy. MRI is superior in its ability to visualize the vagina and cervix and can provide information regarding the thickness of the vaginal septum $[20,30]$.

Though the majority of transverse septa occupy the mid to upper vagina, $14 \%$ are located in the lower vagina. These may be difficult to differentiate from an imperforate hymen or vaginal agenesis. Appropriate classification of the anomaly is critical as the management of each is markedly different. As previously noted, individuals with an imperforate hymen will demonstrate a further bulge of the hymen during Valsalva. A transverse vaginal septum will not demonstrate this mobility. A rectal exam may also be performed to distinguish an obstructive process from vaginal atresia. Those patients with a distal obstruction will have a palpable vaginal bulge on rectal exam due to accumulation and distension of the vagina with menstrual fluid; patients with vaginal atresia will not have such a bulge. As previously noted, ultrasound and/or MRI may be required to confirm the diagnosis and guide surgical management $[20,23]$.

Surgical excision of the transverse vaginal septum is the standard of care. Excision is easiest to perform when a hematocolpos is present and the septum is thin. Complete excision of the septum with reanastomosis of the vaginal mucosa on either sides of the excision is required to prevent vaginal stenosis. Using a spinal needle, the vaginal septum is pierced and the obstructed menstrual fluid is aspirated, confirming that the operator has entered the region of the vagina cephalad to the obstruction. Ultrasound guidance may also be useful. The vaginal septum should then be further excised, to allow for passage of a foley catheter into the upper vagina. This allows maintenance of access to the upper vagina and helps to delineate the boundaries of the septum. The presence of a preoperative hematocolpos provides distention of the vagina, the redundant mucosa of which facilitates reanastomosis of the vaginal mucosa [20].

Alternatively, the "Z-plasty" technique may be utilized for thicker septa or where there is not enough normal mucosa to reapproximate end to end [31]. In this procedure, the mucosa lining the inferior aspect of the septum is incised by two incisions, creating four triangular flaps. The intervening fibrous tissue that comprises the septum is then excised. The superior mucosal edge is then incised in a similar fashion, creating four additional triangular flaps of vaginal mucosa. When creating this second incision, care should be taken to rotate the position of the incision by 45 degrees relative to the incision in the inferior mucosa to allow for the resulting flaps to interdigitate. The upper and lower flaps are then undermined to allow for anastomosis and the free edges of the upper and lower triangular flaps are sewn together to form a $\mathrm{Z}$ plasty. 
Because of the risk of postoperative vaginal strictures, patients should be prepared to use dilators postoperatively [31,32]. If there is concern for patient compliance with postoperative vaginal dilation, excision may be delayed until the patient is mature enough to use dilators. In these scenarios, suppression of menstruation is warranted. It may be tempting to simply drain the hematometrocolpos as a means of postponing surgery. This should be avoided as a microperforation can allow for ascending infections.

\section{Obstructive hemi-vagina with ipsilateral renal anomaly}

Recent attention has been paid to the coexistence of a uterine didelphys, a portion of which is obstructed by a longitudinal vaginal septum, and ipsilateral renal anomaly. The diagnosis of this syndrome, termed obstructive hemi-vagina with ipsilateral renal anomaly (OHVIRA) [33], is often delayed. Dysmenorrhea does not necessarily develop until there has been significant distension of the obstructed hemivagina and uterine horn. In addition, it is not uncommon for patients with this syndrome to be misdiagnosed by their primary provider as demonstrated in a series in which the actual diagnosis had been missed in $100 \%$ of referred cases [34]. In a recent cohort of women with anomalies, confirmation of the diagnosis of OHVIRA was made, on average, 27 months after the initial presentation of symptoms, compared to one month after the onset of symptoms in those patients with a transverse vaginal septum [35].

Patients with OHVIRA will commonly present with initially cyclic and eventually constant pelvic pain [20]. On physical exam, the obstructed hemivagina may be visualized as a bulge in the vaginal sidewall [33]. Though uterine didelphys is the most common uterine anomaly appreciated with the syndrome, other uterine variants such as the septated uterus, a single uterus with two cervices, and bicornuate uterus have been described in this syndrome [36-38]. As with other anomalies, preoperative imaging with ultrasound and MRI are the preferred imaging modalities to diagnose and characterize OHVIRA [7, 8].

The standard of care for OHVIRA is a single-stage excision of the obstructing longitudinal vaginal septum and evacuation of hematometrocolpos [33]. Subsequent pregnancies have been observed in previously obstructed uterine horns [37, 39-42], underscoring the potential benefit of preservation of the previously obstructed uterine horn. Techniques utilized in aiding excision of the transverse vaginal septum such as the use of ultrasound guidance, needle aspiration, and foley catheter may also prove useful in assisting excision of the obstructing longitudinal septum [43]. As with other obstructive anomalies, decompressive transvaginal needle aspiration of the hematocolpos is contraindicated. MRKH and CAIS, or it may occur in patients with normal Müllerian structures. These latter patients will also present with primary amenorrhea but will often have pain due to the obstruction of cervical secretions and/or menstrual fluid. A rectal exam may be useful in palpating the upper vagina which will likely be distended due to obstruction. If the diagnosis is not confirmed on exam, a pelvic ultrasound or MRI may be necessary to evaluate for the presence or absence of Müllerian structures. In those patients 
with isolated vaginal atresia, the atretic portion of the vagina can be excised from a perineal approach with or without laparoscopy [20, 44, 45]. Ideally, the procedure is performed when a hematocolpos is present as it serves as a natural tissue expander and allows for the mucosa of the upper vagina to be mobilized and brought to the perineum [20]. Postoperatively, patients are at variable risks of stricture formation and may benefit from postoperative use of a vaginal dilator [44].

\section{Non-obstructive anomalies of the female reproductive tract}

- Unicornuate Uterus

- Septate Uterus

- Bicornuate Uterus

- Uterine Didelphys

The non-obstructive anomalies of the female reproductive tract are often incidentally found or identified in women with obstetrical complications ranging from recurrent pregnancy loss, fetal malposition, and preterm labor. The incidence of these anomalies is approximately $2 \%$ in the general population but may be as high as $6-7 \%$ in those women with a history of recurrent pregnancy loss [2].

The unicornuate uterus results from failure of one of the Müllerian ducts to develop, resulting in a smaller, deviated cavity, commonly with a single fallopian tube [2]. It may be accompanied by a rudimentary uterine horn which may or may not be communicating. Removal of a non-communicating horn is indicated due to pain associated with menses and the possibility of ectopic pregnancy. This uterine malformation is associated with poor obstetrical outcomes including cervical insufficiency, preterm birth, growth restriction and stillbirth. Live birth rates are low for those women with a unicornuate uterus, ranging from $29.2 \%$ in one cohort to $49.9 \%$ in a recent review of the literature [46].

The septate uterus is the most common Müllerian duct abnormality and is the uterine anomaly most commonly associated with poor obstetric outcomes [2]. In this variant, the two Müllerian ducts have fused but there has been incomplete resorption of the intervening septum. Though hysteroscopic resection of the septum may improve obstetric outcomes [47], no randomized control trials have been performed to date to support the therapeutic benefit of metroplasty [48].

Partial fusion of Müllerian ducts during embryogenesis results in the bicornuate uterus which is characterized by two uterine horns, joined to a single cervix and a single vagina. Similarly to the unicornuate uterus, the bicornuate uterus is associated with an increased miscarriage rate. Unification procedures are possible but should be reserved for those with wellestablished poor obstetric outcomes [2]. 
Uterine didelphys results from complete failure in the medial fusion of the Müllerian ducts, the result of which is a duplicated system with two uterine horns and two cervices. Approximately $75 \%$ of these patients will have a concomitant longitudinal septum which may or may not be symptomatic. Women with a transverse longitudinal septum who report dyspareunia or an inability to control menstrual flow with a tampon benefit from surgical excision of the septum [20].

\section{Compliance with Ethical Standards}

\section{Conflict of Interest}

Janeen L. Arbuckle declares that she has no conflict of interest. Kimberly H. Hoover declares that she has no conflict of interest.

Human and Animal Rights and Informed Consent

This article does not contain any studies with human or animal subjects performed by any of the authors.

\section{References and Recommended Reading}

1. Francis-West GSSBPBP. Larsen's human embryology. Philadelphia: Churchill Livingstone; 2015.

2. Leon Speroff MD, Marc Fritz MD. Clinical gynecologic endocrinology and infertility. Philadelphia: Lippincott Williams \& Wilkins; 2005.

3. The American Fertility Society classifications of adnexal adhesions, distal tubal occlusion, tubal occlusion secondary to tubal ligation, tubal pregnancies, mullerian anomalies and intrauterine adhesions. Fertil Steril. 1988; 49:944-955.

4. Oppelt P, Renner SP, Brucker S, Strissel PL, Strick R, Oppelt PG, et al. The VCUAM (Vagina Cervix Uterus Adnex-associated Malformation) classification: a new classification for genital malformations. Fertil Steril. 2005; 84:1493-7.

5. Grimbizis GF, Campo R. Clinical approach for the classification of congenital uterine malformations. Gynecol Surg. 2012;9:119-29.

6. Grimbizis GF, Gordts S, Di Spiezio Sardo A, Brucker S, De Angelis C, Gergolet M, et al. The ESHRE-ESGE consensus on the classification of female genital tract congenital anomalies. Gynecol Surg. 2013;10:199212.

7. Robbins JB, Broadwell C, Chow LC, Parry JP, Sadowski EA. Mullerian duct anomalies: embryological development, classification, and MRI assessment. J Magn Reson Imaging. 2015;41:1-12.

8. Santos XM, Krishnamurthy R, Bercaw-Pratt JL, Dietrich JE. The utility of ultrasound and magnetic resonance imaging versus surgery for the characterization of mullerian anomalies in the pediatric and adolescent population. J Pediatr Adolesc Gynecol. 2012;25:181-4.

9. Dietrich JE, Millar DM, Quint EH. Non-obstructive mullerian anomalies. J Pediatr Adolesc Gynecol. 2014;27:386-95.

10. Oppelt P, Renner SP, Kellermann A, Brucker S, Hauser GA, Ludwig KS, et al. Clinical aspects of MayerRokitansky-Kuester-Hauser syndrome: recommendations for clinical diagnosis and staging. Hum Reprod. 2006;21:792-7.

11. Committee opinion: no. 562: mullerian agenesis: diagnosis, management, and treatment. Obstet Gynecol. 2013; 121:1134-1137.

12. Evans TN, Poland ML, Boving RL. Vaginal malformations. Am J Obstet Gynecol. 1981;141:910-20.

13. Mongan NP, Tadokoro-Cuccaro R, Bunch T, Hughes IA. Androgen insensitivity syndrome. Best Pract Res Clin Endocrinol Metab. 2015;29:569-80.

14. Oakes MB, Eyvazzadeh AD, Quint E, Smith YR. Complete androgen insensitivity syndrome-a review. J Pediatr Adolesc Gynecol. 2008;21:305-10.

15. Lee PA, Houk CP, Ahmed SF, Hughes IA. Consensus statement on management of intersex disorders. Int Consensus Conf Intersex Pediatr. 2006;118:e488-500.

16. Bean EJ, Mazur T, Robinson AD. Mayer-RokitanskyKuster-Hauser syndrome: sexuality, psychological effects, and quality of life. J Pediatr Adolesc Gynecol. 2009;22:339-46. 
17. Gargollo PC, Cannon Jr GM, Diamond DA, Thomas P, Burke V, Laufer MR. Should progressive perineal dilation be considered first line therapy for vaginal agenesis? J Urol. 2009;182:1882-9.

18. Edmonds DK, Rose GL, Lipton MG, Quek J. MayerRokitansky-Kuster-Hauser syndrome: a review of 245 consecutive cases managed by a multidisciplinary approach with vaginal dilators. Fertil Steril. 2012;97:686-90.

19. Callens N, De Cuypere G, De Sutter P, Monstrey S, Weyers S, Hoebeke P, et al. An update on surgical and non-surgical treatments for vaginal hypoplasia. Hum Reprod Update. 2014;20:775-801.

20. Miller RJ, Breech LL. Surgical correction of vaginal anomalies. Clin Obstet Gynecol. 2008;51:223-36.

21. Patel V, Casey RK, Gomez-Lobo V. Timing of gonadectomy in patients with complete androgen insensitivity syndrome- current recommendations and future directions. J Pediatr Adolesc Gynecol. 2015;28:00167-9.

22. Brannstrom $\mathrm{M}$, Johannesson $\mathrm{L}$, Bokstrom $\mathrm{H}$, Kvarnstrom N, Molne J, Dahm-Kahler P, et al. Livebirth after uterus transplantation. Lancet. 2015;385:607-16.

23. Dietrich JE, Millar DM, Quint EH. Obstructive reproductive tract anomalies. J Pediatr Adolesc Gynecol. 2014;27:396-402.

24. Current evaluation of amenorrhea. Fertil Steril. 2008;90:038.

25. Ahmed S, Morris LL, Atkinson E. Distal mucocolpos and proximal hematocolpos secondary to concurrent imperforate hymen and transverse vaginal septum. J Pediatr Surg. 1999;34:1555-6.

26. Deligeoroglou E, Deliveliotou A, Makrakis E, Creatsas G. Concurrent imperforate hymen, transverse vaginal septum, and unicornuate uterus: a case report. J Pediatr Surg. 2007;42:1446-8.

27. Fedele L, Frontino G, Motta F, Restelli E. A uterovaginal septum and imperforate hymen with a double pyocolpos. Hum Reprod. 2012;27:1637-9.

28. Koyama-Sato M, Hashida O, Nakamura T, Hirahara F, Sakakibara H. Case of early postoperative adhesion in a patient with molimina due to transverse vaginal septum concomitant with imperforate hymen. J Obstet Gynaecol Res. 2015;41:1141-4.

29. Breech LL, Laufer MR. Mullerian anomalies. Obstet Gynecol Clin North Am. 2009;36:47-68.

30. Williams CE, Nakhal RS, Hall-Craggs MA, Wood D, Cutner A, Pattison SH, et al. Transverse vaginal septae: management and long-term outcomes. BJOG.

2014;121:1653-8.

31. Wierrani F, Bodner K, Spangler B, Grunberger W. "Z"plasty of the transverse vaginal septum using Garcia's procedure and the Grunberger modification. Fertil Steril. 2003;79:608-12.

32. Lacy J, Correll GR, Walmer DK, Price TM. Simple vaginal mold for use in the postoperative care of patients with a transverse vaginal septum. Fertil Steril.

2007;87:1225-6.
33. Smith NA, Laufer MR. Obstructed hemivagina and ipsilateral renal anomaly (OHVIRA) syndrome: management and follow-up. Fertil Steril. 2007;87:918-22.

34. Zurawin RK, Dietrich JE, Heard MJ, Edwards CL. Didelphic uterus and obstructed hemivagina with renal agenesis: case report and review of the literature. J Pediatr Adolesc Gynecol. 2004;17:137-41.

35. Joki-Erkkila MM, Heinonen PK. Presenting and longterm clinical implications and fecundity in females with obstructing vaginal malformations. J Pediatr Adolesc Gynecol. 2003;16:307-12.

36. Shah DK, Laufer MR. Obstructed hemivagina and ipsilateral renal anomaly (OHVIRA) syndrome with a single uterus. Fertil Steril. 2011;96:24.

37. Haddad B, Barranger E, Paniel BJ. Blind hemivagina: long-term follow-up and reproductive performance in 42 cases. Hum Reprod. 1999; 14:1962-4.

38. Fedele L, Motta F, Frontino G, Restelli E, Bianchi S. Double uterus with obstructed hemivagina and ipsilateral renal agenesis: pelvic anatomic variants in 87 cases. Hum Reprod. 2013;28:1580-3.

39. Altchek A, Paciuc J. Successful pregnancy following surgery in the obstructed uterus in a uterus didelphys with unilateral distal vaginal agenesis and ipsilateral renal agenesis: case report and literature review. J Pediatr Adolesc Gynecol. 2009;22:3.

40. Tong J, Zhu L, Lang J. Clinical characteristics of 70 patients with Herlyn-Werner-Wunderlich syndrome. Int J Gynaecol Obstet. 2013;121:173-5.

41. Heinonen PK. Clinical implications of the didelphic uterus: long-term follow-up of 49 cases. Eur J Obstet Gynecol Reprod Biol. 2000;91:183-90.

42. Heinonen PK. Pregnancies in women with uterine malformation, treated obstruction of hemivagina and ipsilateral renal agenesis. Arch Gynecol Obstet. 2013;287:975-8.

43. Candiani GB, Fedele L, Candiani M. Double uterus, blind hemivagina, and ipsilateral renal agenesis: 36 cases and long-term follow-up. Obstet Gynecol. 1997;90:26-32.

44. Mansouri R, Dietrich JE. Postoperative course and complications after pull-through vaginoplasty for distal vaginal atresia. J Pediatr Adolesc Gynecol. 2015;28:433-6.

45. Ciftci I. Laparoscopic-assisted perineal pull-through vaginoplasty. J Pediatr Surg. 2012;47:070.

46. Reichman D, Laufer MR, Robinson BK. Pregnancy outcomes in unicornuate uteri: a review. Fertil Steril. 2009;91:1886-94.

47. Paradisi R, Barzanti R, Fabbri R. The techniques and outcomes of hysteroscopic metroplasty. Curr Opin Obstet Gynecol. 2014;26:295-301.

48. Kowalik CR, Goddijn M, Emanuel MH, Bongers MY, Spinder T, de Kruif JH, Mol BW, Heineman MJ. Metroplasty versus expectant management for women with recurrent miscarriage and a septate uterus. Cochrane Database Syst Rev. 2011;15. 